\title{
Tensor-network Study of Correlation-spreading Dynamics in the Two-dimensional Bose-Hubbard Model
}

Ryui Kaneko ( $\square$ rkaneko@phys.kindai.ac.jp)

Kindai University https://orcid.org/0000-0001-7994-6381

Ippei Danshita

Kyoto University https://orcid.org/0000-0003-4579-2163

\section{Article}

Keywords: Tensor-network study, two-dimensional Bose-Hubbard model, correlation-spreading dynamics, analog quantum, cold atoms and trapped ions, experiments

Posted Date: October 18th, 2021

DOI: https://doi.org/10.21203/rs.3.rs-952110/v1

License: (c) (i) This work is licensed under a Creative Commons Attribution 4.0 International License.

Read Full License

Version of Record: A version of this preprint was published at Communications Physics on March 21st, 2022. See the published version at https://doi.org/10.1038/s42005-022-00848-9. 
1 Tensor-network study of correlation-spreading dynamics in the two-dimensional Bose-Hubbard model

\author{
Ryui Kaneko* and Ippei Danshita ${ }^{\dagger}$ \\ Department of Physics, Kindai University, \\ Higashi-Osaka, Osaka 577-8502, Japan
}

(Dated: September 30, 2021) 


\begin{abstract}
Recent developments in analog quantum simulators based on cold atoms and trapped ions call for cross-validating the accuracy of quantum-simulation experiments with use of quantitative numerical methods; however, it is particularly challenging for dynamics of systems with more than one spatial dimension. Here we demonstrate that a tensor-network method running on classical computers is useful for this purpose. We specifically analyze real-time dynamics of the two-dimensional BoseHubbard model after a sudden quench starting from the Mott insulator by means of the infinite projected entangled pair state algorithm. Calculated single-particle correlation functions are found to be in good agreement with a recent experiment [Y. Takasu et al., Sci. Adv. 6, eaba9255 (2020)]. By estimating the phase and group velocities from the single-particle and density-density correlation functions, we predict how these velocities vary in the moderate interaction region, which serves as a quantitative benchmark for future experiments.
\end{abstract}

\title{
7 I. INTRODUCTION
}

8 State-of-art experimental platforms of cold atoms and trapped ions as analog quantum 9 simulators have offered unique opportunities for studying far-from-equilibrium dynamics of 10 isolated quantum many-body systems. Thanks to their high controllability and long co11 herence time, these platforms have already addressed a variety of intriguing phenomena 12 that are in general difficult to simulate with classical computers, such as correlation spread${ }_{13}$ ing [1-3] and relaxation [4-6] after a quantum quench, many-body localization in a disorder 14 potential [7-9], and quantum scar states [10, 11]. Nevertheless, accurate numerical meth15 ods using classical computers are highly demanded at the current stage of the studies of 16 quantum many-body dynamics, since the classical computation still has complementary ad17 vantages over the quantum simulation in that it is free of noise and much more accessible 18 owing to its wide dissemination. In this sense, it is important to cross-check the validity 19 of quantum-simulation experiments and some numerical methods by comparing them with 20 each other.

${ }_{21}$ In particular, direct comparisons between experimental and numerical outputs have

\footnotetext{
* rkaneko@phys.kindai.ac.jp

$\dagger$ danshita@phys.kindai.ac.jp
} 


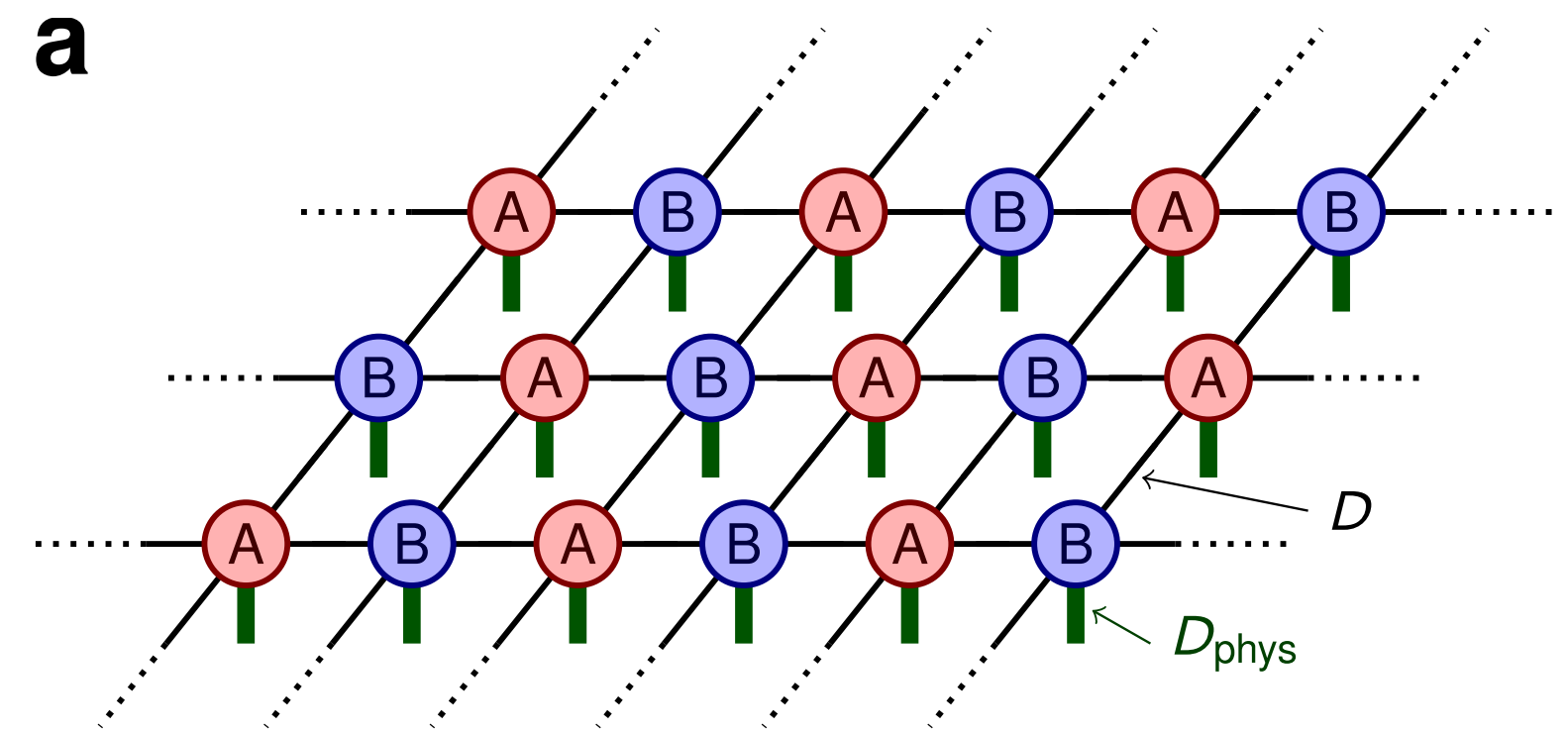

b

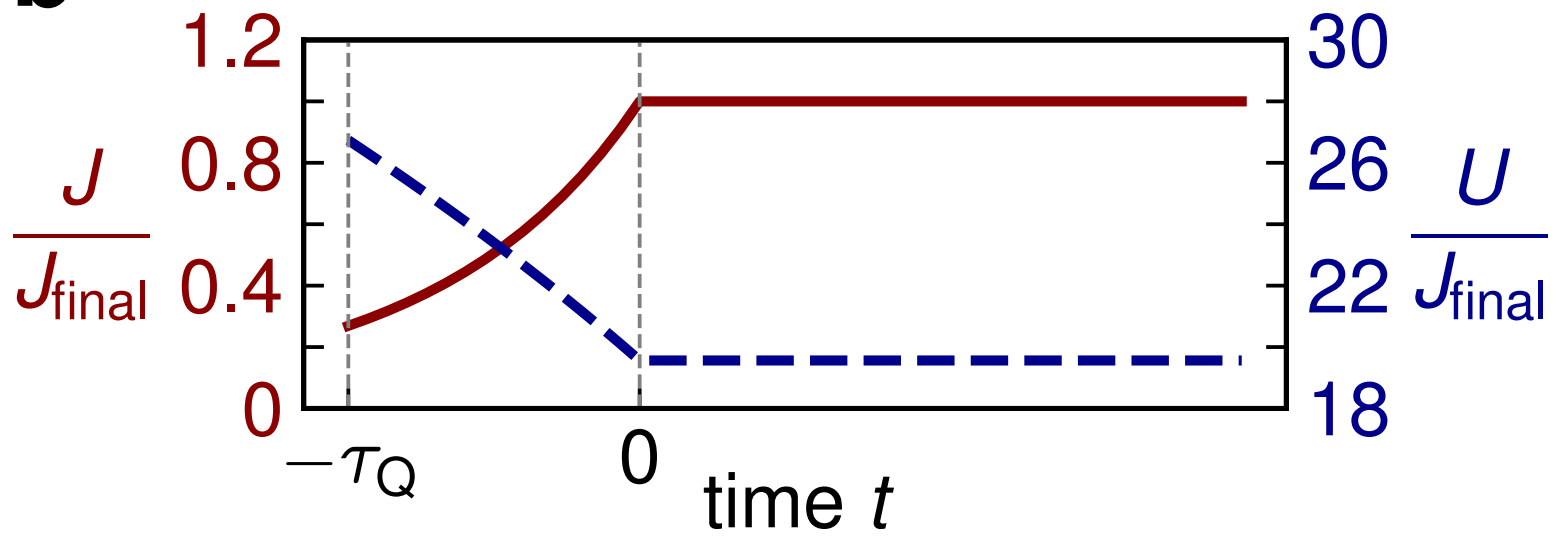

FIG. 1. (a) Schematic figure of iPEPS with a two-site unit cell. A rank-five tensor at each site is represented as a circle with four thin lines and one thick line. The former lines correspond to the virtual degrees of freedom with the bond dimension $D$, while the latter line corresponds to the physical degrees of freedom with the dimension of the local Hilbert space $D_{\text {phys }}$. The wave functions become more accurate as $D$ increases. (b) Time dependence of the hopping $J$ (a red solid line) and the onsite interaction $U$ (a blue dashed line) with a finite-time quench. The parameter $U / J$ is varied from $\sim 99.4$ to $\sim 19.6$ for $-\tau_{\mathrm{Q}}<t<0$. In the case of a sudden quench, we discard the region $-\tau_{\mathrm{Q}}<t<0$.

22 been made for dynamical spreading of two-point spatial correlations of the Bose-Hubbard 23 model [1, 3, 12-15], which can be realized experimentally with ultracold bosons in optical ${ }_{24}$ lattices [16]. The correlation spreading has attracted much theoretical interest [12-15, 17-24] 
${ }_{25}$ in the sense that it is closely related to fundamental phenomena, including the propagation 26 of quantum information and the thermalization. In one spatial dimension, quasi-exact nu${ }_{27}$ merical methods based on matrix product states (MPSs) have been used to validate the ${ }_{28}$ performance of the quantum simulators $[1,3,12]$. In two dimensions (2D), by contrast, 29 accurate numerical simulations are challenging. Indeed, the comparisons with respect to 30 a single-particle correlation have shown that a few types of the truncated Wigner approx31 imation (TWA) fail to capture the real-time evolution accurately enough to extract the 32 propagation velocity of the correlation $[3,14]$. Moreover, while the propagation velocities 33 obtained by a two-particle irreducible strong-coupling (2PISC) approach quantitatively agree 34 with the experimental value, its applicability is limited to a strongly interacting regime, and 35 it does not accurately give the value of the correlation itself [15].

In this paper, we present quantitative numerical analyses of the correlation-spreading ${ }_{37}$ dynamics of the 2D Bose-Hubbard model starting from a Mott insulating initial state with 38 unit filling. To this end, we employ the tensor-network method based on the infinite proз9 jected entangled pair state (iPEPS) [25-32] or the tensor product state [33-37], which is an 40 extension of MPS to 2D systems [see Fig.1(a)]. The iPEPS studies on real-time dynamics of ${ }_{41}$ isolated [38-46] and open [38, 39, 47-49] quantum systems in 2D have begun very recently. 42 Previous simulations suggest that iPEPS can represent relatively low-entangled states in 43 short-time dynamics for simple spin $1 / 2$ systems [38-41] and some itinerant electron sys44 tems [43]. This observation may be valid for real-time dynamics in Bose-Hubbard systems; 45 however, little is known about it until now. We find that the single-particle correlation com${ }_{46}$ puted with iPEPS, as well as the estimated propagation velocity of the correlation front, 47 agrees very well with the experimental result [3], demonstrating that iPEPS can be useful for 48 actual quantum-simulation experiments. We also conduct numerical simulations in a mod${ }_{49}$ erate interaction region, which has not been addressed by the previous experiments $[1,3]$. ${ }_{50}$ From the real-time evolution of the single-particle and density-density correlations, we show ${ }_{51}$ that the phase and group velocities approach each other when the interaction decreases. 


\section{${ }_{52}$ II. RESULTS}

\section{A. Model}

${ }_{54}$ We consider the Bose-Hubbard model on a square lattice [50, 51]. The Hamiltonian is 55 given as

$$
\hat{H}=-J \sum_{\langle i j\rangle}\left(\hat{a}_{i}^{\dagger} \hat{a}_{j}+\hat{a}_{j}^{\dagger} \hat{a}_{i}\right)+\frac{U}{2} \sum_{i} \hat{n}_{i}\left(\hat{n}_{i}-1\right)-\mu \sum_{i} \hat{n}_{i}
$$

${ }_{56}$ where $\hat{a}_{i}^{\dagger}$ and $\hat{a}_{i}$ are the creation and annihilation operators at site $i, \hat{n}_{i}=\hat{a}_{i}^{\dagger} \hat{a}_{i}$ is the number ${ }_{57}$ operator, $J$ is the strength of the hopping between nearest-neighbor sites, $U$ is the strength 58 of the onsite interaction, and $\mu$ is the chemical potential. The notation $\langle i j\rangle$ indicates that ${ }_{59}$ sites $i$ and $j$ are nearest neighbors. For simplicity, we ignore the effects of the trap potential 60 and the Gaussian envelopes of optical lattice lasers, which do not affect short-time dynamics. ${ }_{61}$ We set the lattice spacing $d_{\text {lat }}$ to be unity. The ground state at the commensurate filling is 62 the Mott insulating (superfluid) state for $U \gg J(U \ll J)$. Hereafter, we will consider a 63 sudden quench and a quench with a short time [see Fig. 1(b)]. 65 nalization and the experiment

66 Let us first focus on the case of a sudden quench. We compare our results of iPEPS with 67 those of the exact diagonalization (ED) method and obtain consistent results in a short time. ${ }_{68}$ In the ED simulations using the QuSpin library $[52,53]$, we choose the system sizes $L_{x} \times L_{y}$ 69 up to $5 \times 4$ and use the periodic-periodic boundary condition. We examine to what extent 70 the energy is conserved in the iPEPS simulations. The grand potential density $\langle\hat{H}\rangle$ at $T=0$ ${ }_{71}$ starting from the Mott insulator $\otimes_{i}\left|n_{i}=1\right\rangle$ should remain constant. They well converge 72 for $D \geq 6$ and remain nearly constant up to $t \sim 0.4 \hbar / J[54]$. We also investigate how 73 the single-particle correlations converge with increasing bond dimensions. The equal-time ${ }_{74}$ single-particle correlation function at a distance $\boldsymbol{r}=(x, y)$ for the system size $N_{\mathrm{s}}$ is defined 75 as

$$
C_{\boldsymbol{r}}^{\mathrm{sp}}(t)=\frac{1}{2 N_{\mathrm{s}}} \sum_{i, j}^{\prime}\left\langle\hat{a}_{i}^{\dagger}(t) \hat{a}_{j}(t)+\hat{a}_{j}^{\dagger}(t) \hat{a}_{i}(t)\right\rangle
$$




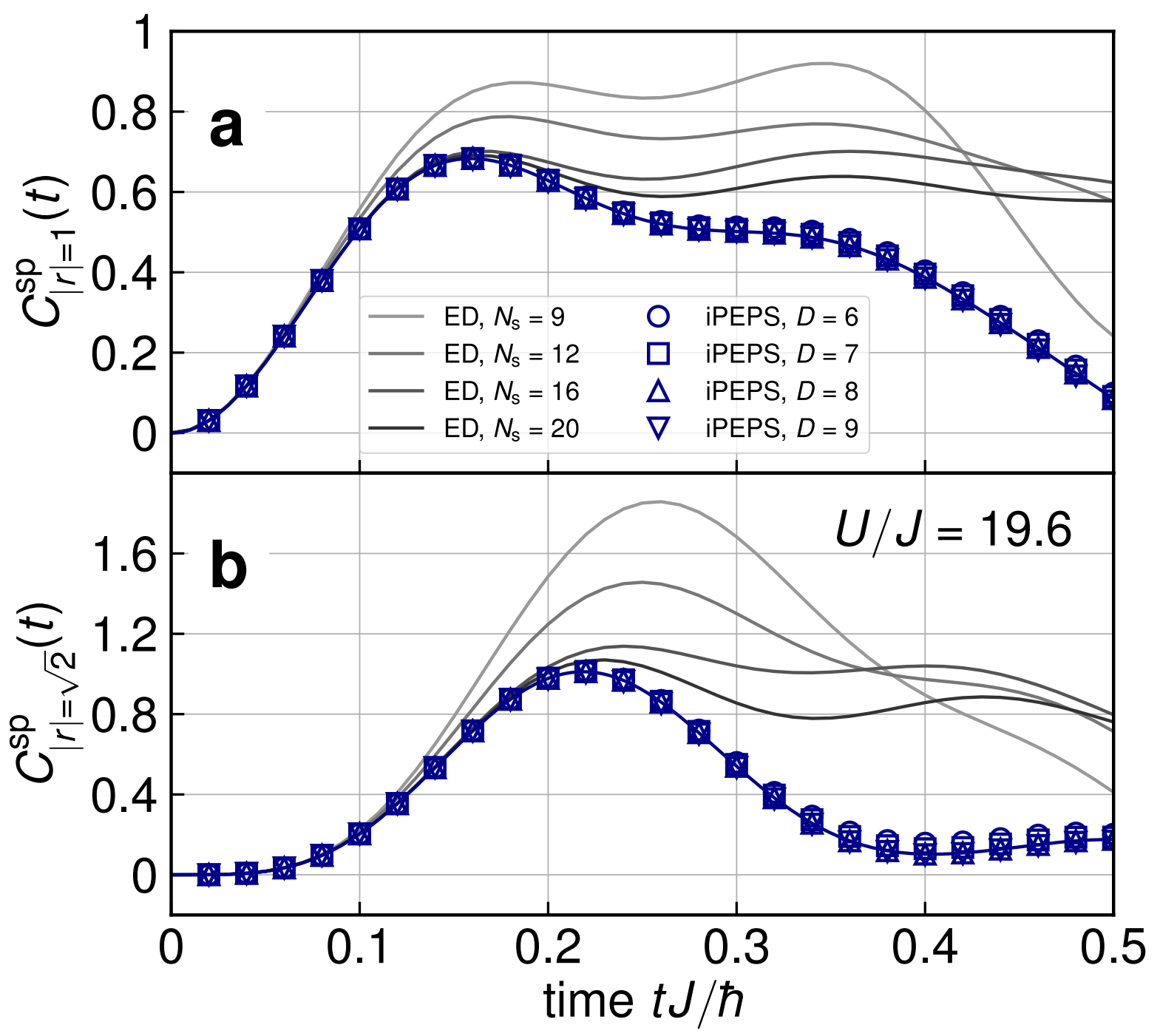

FIG. 2. Comparison of the single-particle correlation functions in the case of a sudden quench between iPEPS (blue lines with symbols) and ED (gray lines). The correlations at distances (a) $|\boldsymbol{r}|=1$ and (b) $|\boldsymbol{r}|=\sqrt{2}$ are shown. Both results overlap perfectly in a short time.

76 Here $\sum_{i, j}^{\prime}$ denotes the summation over $(i, j)$ that satisfies $\left|x_{j}-x_{i}\right|=x$ and $\left|y_{j}-y_{i}\right|=y$. In 77 the iPEPS simulations, $1 / N_{\mathrm{s}} \times \sum_{i, j}^{\prime}$ is replaced by $1 / 2 \times \sum_{i=A, B} \sum_{j}^{\prime}$ with $A$ and $B$ being 78 sublattice sites because of the translational invariance. As shown in Fig. 2, $C_{|\boldsymbol{r}|=1}^{\mathrm{sp}}(t):=$ $79\left[C_{\boldsymbol{r}=(1,0)}^{\mathrm{sp}}(t)+C_{\boldsymbol{r}=(0,1)}^{\mathrm{sp}}(t)\right] / 2$ exhibits a peak at $t \sim 0.15 \hbar / J$ in both results, and they overlap so perfectly in this short time. For $t \gtrsim 0.15 \hbar / J$, the correlation functions of ED start to exhibit s1 a significant finite-size effect, whereas those of iPEPS converge for $D \geq 6$. We observe similar 82 behavior for $C_{|\boldsymbol{r}|=\sqrt{2}}^{\mathrm{sp}}(t):=C_{\boldsymbol{r}=(1,1)}^{\mathrm{sp}}(t)$. The iPEPS results are better simulated up to a longer 83 time. 


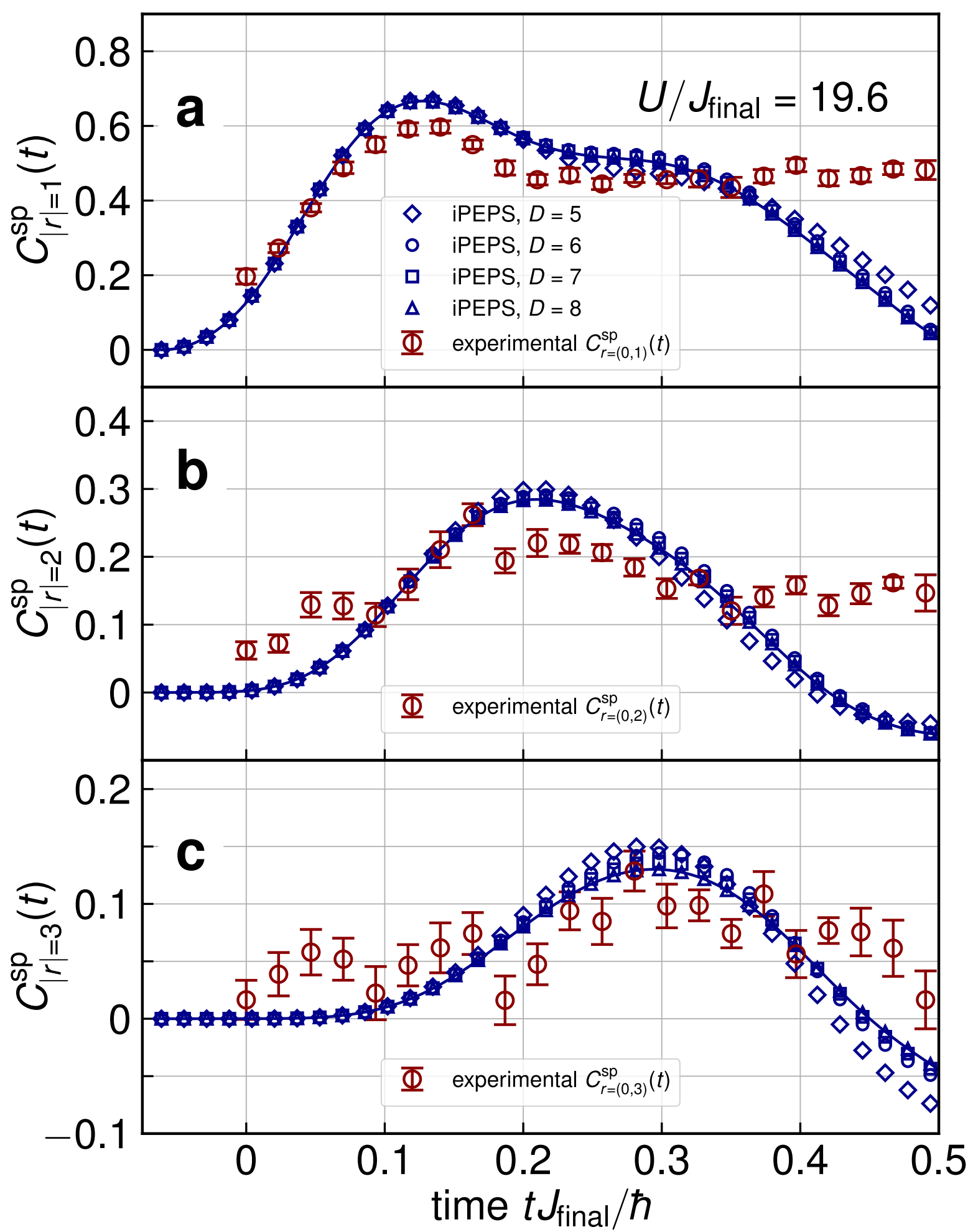

FIG. 3. Comparison of the single-particle correlation functions in the case of a finite-time quench between iPEPS (blue lines with symbols) and the experiment (red circles). The correlations at distances (a) $|\boldsymbol{r}|=1$, (b) $|\boldsymbol{r}|=2$, and (c) $|\boldsymbol{r}|=3$ are shown. Both results are in good agreement. 
Next, we compare the correlations of iPEPS with those of the experiment [3] for a finite 85 quench time. Figures $3(\mathrm{a}-\mathrm{c})$ show the time evolution of correlations at distances $|\boldsymbol{r}|=1,2$, ${ }_{86}$ and 3, respectively. Qualitative behavior is essentially equivalent to the case of the sudden 87 quench, although the correlation function shifts to an earlier time. For $|\boldsymbol{r}|=1$, both data 8 show a peak at $t \sim 0.12 \hbar / J_{\text {final }}$. Similarly, the first-peak times are consistent with each other ${ }_{89}$ for $|\boldsymbol{r}|=2$ and 3, and they become longer with increasing distances. When the energy is 90 approximately conserved (namely, for $t \lesssim 0.4 \hbar / J_{\text {final }}[54]$ ), the intensities of correlations also 91 overlap very well. They are also consistent with those obtained by TWA [3, 13, 14], while ${ }_{92}$ the iPEPS simulations can deal with a slightly longer time and capture the correlation peaks 93 more clearly [54].

\section{C. Estimates of group and phase velocities in the moderate interaction region}

95 Having confirmed the applicability of iPEPS simulations to real-time evolution of the 96 Bose-Hubbard model, we study how information propagates by a sudden quench in the ${ }_{97}$ moderate interaction region. There are two kinds of velocity that are relevant to the cor98 relation spreading. One is the group velocity $v_{\mathrm{gr}}$, which corresponds to the propagation of ${ }_{99}$ the envelope of the wave packet and is a suitable quantity to characterize the spreading of 100 correlations. In non-relativistic quantum many-body systems, $v_{\text {gr }}$ is bounded above, and 101 the upper bound is known as the Lieb-Robinson bound [23, 24, 55, 56]. Notice that the 102 Lieb-Robinson bound for the Bose-Hubbard model has not been rigorously derived with a 103 few exceptions for limited situations $[18,23,24]$. The phase velocity $v_{\mathrm{ph}}$ is the other charac104 teristic quantity, which corresponds to the propagation of the first peak of the wave packet, 105 and does not have to obey the Lieb-Robinson bound.

106 To estimate the group velocity from the single-particle correlations, long-time simulations 107 are required in general. However, it is challenging in the iPEPS simulations. To circumvent 108 the difficulty, we estimate the group velocity by the density-density correlation. It is known 109 that the propagation velocity of the first peak of this correlation agrees very well with the 110 group velocity $[1,12]$. The equal-time density-density correlation function at a distance ${ }_{111} \boldsymbol{r}=(x, y)$ for the system size $N_{\mathrm{s}}$ is defined as

$$
C_{\boldsymbol{r}}^{\mathrm{dd}}(t)=\frac{1}{N_{\mathrm{s}}} \sum_{i, j}^{\prime}\left\langle\hat{n}_{i}(t) \hat{n}_{j}(t)\right\rangle_{c}
$$




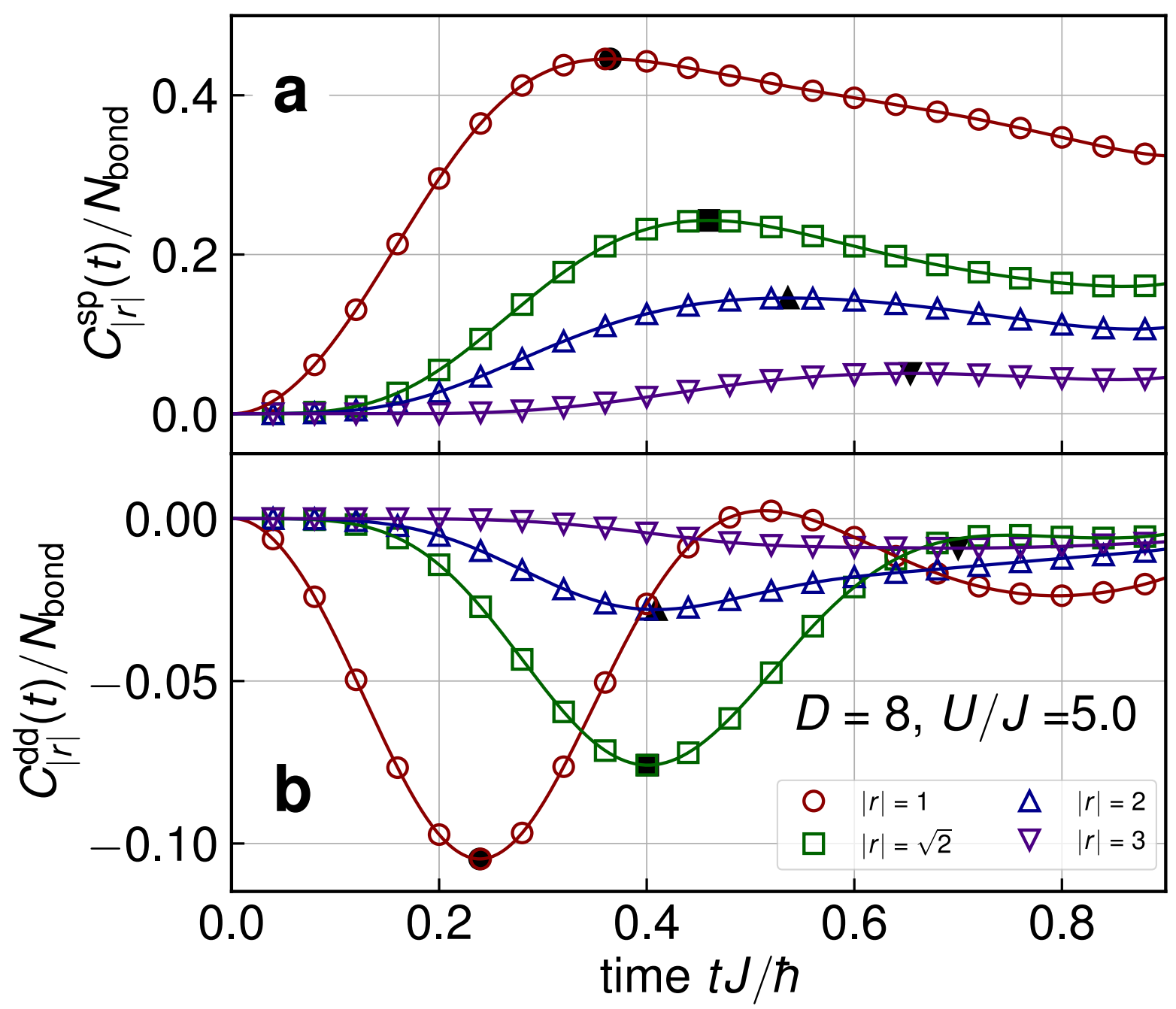

FIG. 4. (a) Single-particle and (b) density-density correlation functions per bond at $U / J=5$ for the bond dimension $D=8$. The normalization factor at a distance $\boldsymbol{r}=(x, y)$ is given as $N_{\text {bond }}=2$ for $x \neq y\left(|\boldsymbol{r}|=1,2\right.$, and 3), while it is $N_{\text {bond }}=4$ for $x=y(|\boldsymbol{r}|=\sqrt{2})$. The black symbol corresponds to the first peak in the correlation function obtained by cubic spline interpolation of data points. The propagation velocities along the horizontal or vertical axis are extracted by the data at $|\boldsymbol{r}|=1,2$, and 3 . The velocity estimated from the density-density correlation functions is slower than that from the single-particle correlation functions.

112 where $\langle\cdots\rangle_{c}$ denotes a connected correlation function. In our simulations, $\left\langle\hat{n}_{i}(t) \hat{n}_{j}(t)\right\rangle_{c}=$ ${ }_{113}\left\langle\hat{n}_{i}(t) \hat{n}_{j}(t)\right\rangle-1$ because $\left\langle\hat{n}_{i}(t)\right\rangle=1$ for all sites and time steps. As in $C_{\boldsymbol{r}}^{\mathrm{sp}}(t)$, the summation 114 is replaced by that within sublattice sites in the iPEPS simulations. The parity-parity 115 correlation closely related to the density-density one can be measured in experiments by 116 using the quantum-gas microscope techniques [1]. 


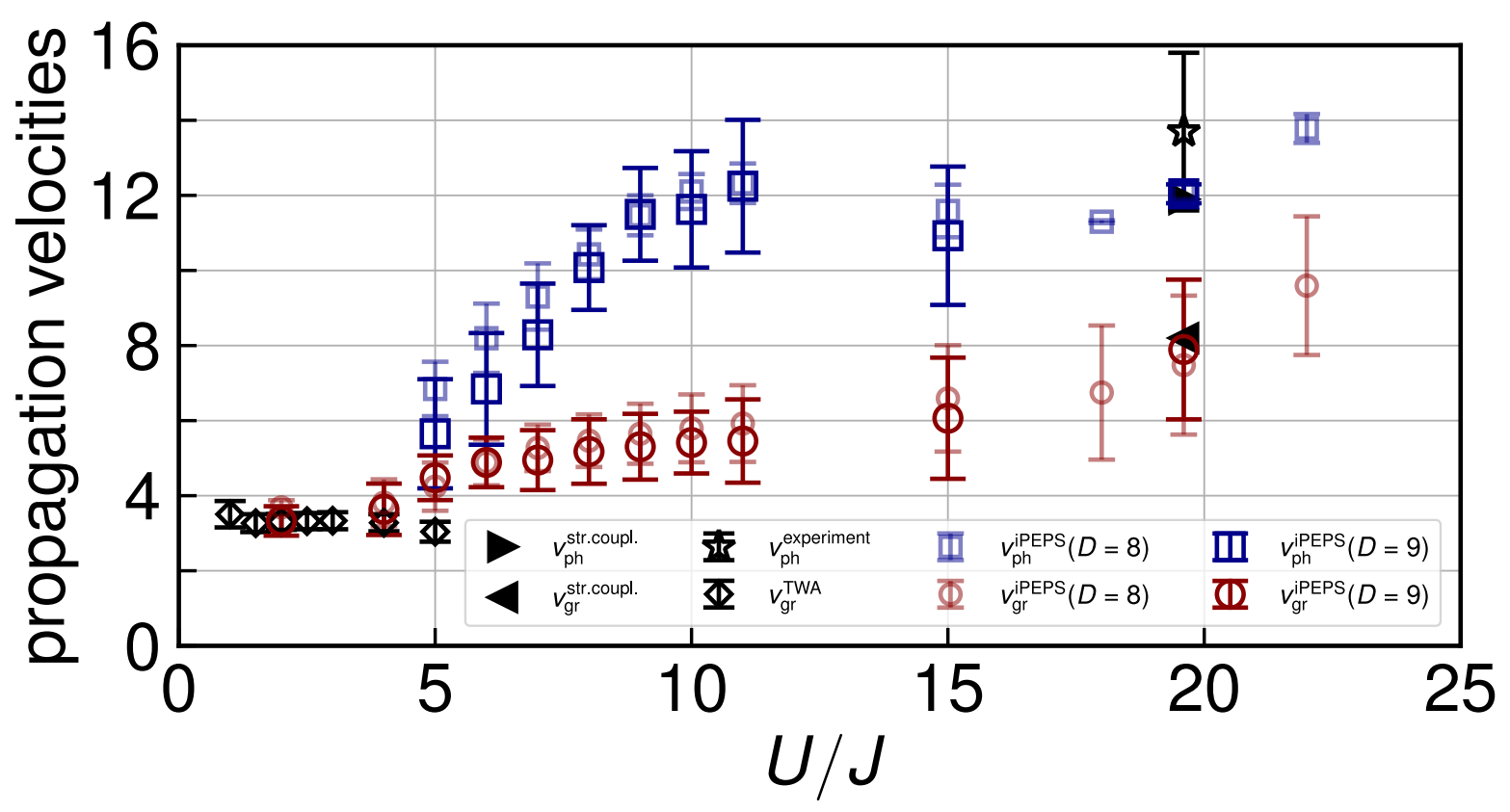

FIG. 5. Estimated group (red circles) and phase (blue squares) velocities from the density-density and single-particle correlation functions for the bond dimensions $D=8$ and $D=9$. The data for $D=8$ and $D=9$ overlap within the error bars. The velocities and their error bars are obtained by extrapolation of the distance dependence of the peak time. The results obtained by the 2PISC approach [15] (triangles), TWA [13] (diamonds), and the experiment [3] (a star) are also shown. Both velocities gradually merge with decreasing interaction.

${ }_{117}$ We extract the propagation velocities from the first peak in both correlations for $|\boldsymbol{r}|=1$, ${ }_{118} 2$, and 3. For simplicity, we consider the sudden quench hereafter. When the interaction 119 becomes weaker, we have confirmed that the energy is conserved in a longer time frame; 120 typically, $t \lesssim 0.9 \hbar / J$ for $U / J \sim 5$ [54]. All the correlation peaks for $|\boldsymbol{r}| \leq 3$ appear in ${ }_{121}$ this time frame (see Fig. 4). The first peak of the single-particle correlation appears at ${ }_{122} t \sim 0.35 \hbar / J$ for $|\boldsymbol{r}|=1$, while it appears at $t \sim 0.65 \hbar / J$ for $|\boldsymbol{r}|=3$. By contrast, the first ${ }_{123}$ peak of the density-density correlation appears at $t \sim 0.25 \hbar / J$ for $|\boldsymbol{r}|=1$, while it appears 124 at $t \sim 0.7 \hbar / J$ for $|\boldsymbol{r}|=3$. It takes a long time for propagation in the latter case. The 125 first-peak time is almost a linear function of the distance [54], and the system exhibits the ${ }_{126}$ light-cone-like spreading of correlations.

${ }_{127}$ We summarize the interaction dependence of the group and phase velocities in Fig. 5. In 128 the weak interaction region, the estimated group velocities are $v_{\mathrm{gr}} \sim 4 J / \hbar$. They are similar ${ }_{129}$ to those obtained by the TWA at filling factor $\nu=10$ [13]. They are also consistent with the 
130 group velocity $v_{\text {gr }}(U=0)=4 J / \hbar$ of a single particle [13]. In the strong interaction region, 131 the estimated group velocity $v_{\mathrm{gr}} \sim(8 \pm 2) \mathrm{J} / \hbar$ at $U / J=19.6$ coincides with that obtained 132 by the 2PISC approach [15] within the error bar of extrapolation. It is also comparable to 133 the group velocity $v_{\mathrm{gr}}(U \gg J)=6 J / \hbar \times\left[1+\mathcal{O}\left(J^{2} / U^{2}\right)\right]$ of a quasi-particle in the large $U$ 134 limit $[1,3,12]$. Similarly, the estimated phase velocity agrees very well with the results of 135 the 2PISC approach [15] and the experiment [3]. In the intermediate region, the estimated 136 group velocity is closer to the single-particle group velocity in the superfluid region, whereas 137 it is comparable to the 2PISC result near and above the critical point $U_{\mathrm{c}} / J \sim 16.7[57-59]$. ${ }_{138}$ Both group and phase velocities gradually converge to the same value as $U / J$ is decreased.

\section{III. CONCLUSION}

We have studied real-time dynamics of the 2D Bose-Hubbard model after a sudden quench 141 starting from the Mott insulator with unit filling. We have employed the 2D tensor-network 142 method based on the infinite projected entangled pair states, which are the 2D extension of 143 the well-known matrix product states in one dimension. Calculated single-particle correla144 tion functions reproduce the recent experimental results very well. The iPEPS algorithm can 145 simulate real-time dynamics long enough for extracting the propagation velocities from cor146 relations. We have also estimated the group and phase velocities in the moderate interaction 147 region, in which the 2PISC approach and the TWA are not applicable. Our findings would ${ }_{148}$ be useful in the future analog quantum simulation of Bose-Hubbard systems. The ability of 149 the tensor-network method that accurately calculates the real-time dynamics of 2D quan150 tum many-body systems opens up the possibility of applying it to other quantum-simulation 151 platforms, such as Rydberg atoms, trapped ions, and superconducting circuits.

\section{IV. METHODS}

\section{A. Real-time evolution by infinite projected entangled pair states}

${ }_{154}$ We prepare iPEPS with a two-site unit cell [see Fig. 1(a)]. The symbols $D$ and $D_{\text {phys }}$ 155 denote the virtual bond dimension and the dimension of the local Hilbert space, respectively. ${ }_{156}$ The former improves the accuracy of the wave function, whereas the latter corresponds to ${ }_{157}$ the maximum particle number $n_{\max }$ as $D_{\text {phys }}=n_{\max }+1$. Although $n_{\max }$ can take infinity in 
158 Bose-Hubbard systems, it is practically bounded above in the presence of interaction [60, 61]. ${ }_{159}$ We can choose finite $D_{\text {phys }}$ in the simulations of real-time dynamics. In the case of a sudden 160 quench to the Mott insulating region $\left(U / J>U_{\mathrm{c}} / J \sim 16.7\right.$ [57-59]), we set the dimension of 161 the local Hilbert space as $D_{\text {phys }}=3$ because the number of particles deviates only slightly 162 from unity $[60,61]$. For $U / J<U_{\mathrm{c}} / J$, we choose $D_{\text {phys }}=5$ so that the wave functions can ${ }_{163}$ further take into account the effect of particle fluctuations. When $U$ is close to zero (at ${ }_{164} U / J=2$ in our simulations), we use slightly larger $D_{\text {phys }}=7$. The initial Mott insulating 165 state $\otimes_{i}\left|n_{i}=1\right\rangle$ can be represented with the bond dimension $D=1$. As for static properties, 166 the Bose-Hubbard model was investigated by finite PEPS or iPEPS, and the phase transition 167 between the Mott insulating and superfluid phases was reproduced [62-70].

${ }_{168}$ The wave function at each time $|\Psi(t)\rangle=e^{-i t \hat{H} / \hbar}|\Psi(0)\rangle$ is obtained by real-time evolving 169 iPEPS [38-40]. The real-time evolution operator in a small time step $d t$ can be approximated 170 by the Suzuki-Trotter decomposition [71-73] as $e^{-i d t \hat{H} / \hbar} \sim \prod_{\langle i j\rangle} e^{-i d t \hat{H}_{i j} / \hbar}$, where $\hat{H}_{i j}=$ ${ }_{171}-J\left(\hat{a}_{i}^{\dagger} \hat{a}_{j}+\hat{a}_{j}^{\dagger} \hat{a}_{i}\right)+U\left[\hat{n}_{i}\left(\hat{n}_{i}-1\right)+\hat{n}_{j}\left(\hat{n}_{j}-1\right)\right] /(2 z)-\mu\left(\hat{n}_{i}+\hat{n}_{j}\right) / z$ with the coordination 172 number $z=4$ is the local Hamiltonian satisfying $\hat{H}=\sum_{\langle i j\rangle} \hat{H}_{i j}$. After applying the two173 site gate $e^{-i d t \hat{H}_{i j} / \hbar}$ to neighboring tensors, we approximate the local tensors by the singular 174 value decomposition in such a way that the virtual bond dimension of iPEPS remains $D$. 175 In the actual simulations, the second-order Suzuki-Trotter decomposition is used for this 176 simple update algorithm [29, 74], and the tensor-network library TeNeS [75, 76] is adopted. 177 The wave functions are optimized up to the bond dimension $D=9$. As we will see later, 178 qualitative behavior of correlation functions is nearly the same for $D \geq 6$. When extracting 179 the propagation velocities, we mainly use the data for $D=8$ and $D=9$ to ensure sufficient 180 convergence of physical quantities. We have numerically found that at these values of $D$, 181 the number of particles is nearly conserved during the real-time evolution starting from the 182 Mott insulator.

183 Physical quantities in the thermodynamic limit are calculated by the corner transfer 184 matrix renormalization group (CTMRG) method [30-32, 34, 77-83]. The bond dimension 185 of the environment tensors is chosen as $\chi=2 D^{2}$ to ensure that physical quantities are well 186 converged.

${ }_{187}$ To compare our results obtained by iPEPS with the experiment [3], we consider a quench ${ }_{188}$ with a short time $\tau_{\mathrm{Q}}=0.1 \mathrm{~ms}[13,14]$ [see Fig. 1(b)]. For $-\tau_{\mathrm{Q}}<t<0$, both $J$ and $U$ are 189 controlled. The wave function is updated as $|\Psi(t+d t)\rangle \sim e^{-i d t \hat{H}(t) / \hbar}|\Psi(t)\rangle$ with the time- 
190 dependent Hamiltonian $\hat{H}(t)$ in this region. For $t>0$, both parameters are fixed. We take ${ }_{191} J_{\text {final }}=J(t=0) \sim 0.0612 \hbar / \tau_{\mathrm{Q}}$ as the unit of energy. The discrete time step for the real-time 192 evolution is set to be $d t /\left(\hbar / J_{\text {final }}\right)=\tau_{\mathrm{Q}} /\left(\hbar / J_{\text {final }}\right) / 15 \sim 0.00408$ for all $t$. To compare the 193 iPEPS results with the exact real-time dynamics in finite-size systems, we also consider a ${ }_{194}$ sudden parameter change and set the time step as $d t /(\hbar / J)=0.005$. We have checked that 195 the simulations with doubled and halved $d t$ do not change the results significantly.

${ }_{196}$ V. DATA AVAILABILITY

The data in this paper are available from the authors upon request.

VI. CODE AVAILABILITY

The codes in this paper are available from the authors upon request.

[1] M. Cheneau, P. Barmettler, D. Poletti, M. Endres, P. Schauß, T. Fukuhara, C. Gross, I. Bloch, C. Kollath, and S. Kuhr, Light-cone-like spreading of correlations in a quantum many-body system, Nature 481, 484 (2012).

[2] P. Jurcevic, B. P. Lanyon, P. Hauke, C. Hempel, P. Zoller, R. Blatt, and C. F. Roos, Quasiparticle engineering and entanglement propagation in a quantum many-body system, Nature 511, 202 (2014).

[3] Y. Takasu, T. Yagami, H. Asaka, Y. Fukushima, K. Nagao, S. Goto, I. Danshita, and Y. Takahashi, Energy redistribution and spatio-temporal evolution of correlations after a sudden quench of the bose-hubbard model, Sci. Adv. 6, eaba9255 (2020).

[4] S. Trotzky, Y.-A. Chen, A. Flesch, I. P. McCulloch, U. Schollwöck, J. Eisert, and I. Bloch, Probing the relaxation towards equilibrium in an isolated strongly correlated one-dimensional bose gas, Nat. Phys. 8, 325 (2012).

[5] T. Langen, S. Erne, R. Geiger, B. Rauer, T. Schweigler, M. Kuhnert, W. Rohringer, I. E. Mazets, T. Gasenzer, and J. Schmiedmayer, Experimental observation of a generalized gibbs ensemble, Science 348, 207 (2015). 
[6] A. M. Kaufman, M. E. Tai, A. Lukin, M. Rispoli, R. Schittko, P. M. Preiss, and M. Greiner, Quantum thermalization through entanglement in an isolated many-body system, Science $\mathbf{3 5 3}$, $794(2016)$.

[7] M. Schreiber, S. S. Hodgman, P. Bordia, H. P. Lüschen, M. H. Fischer, R. Vosk, E. Altman, U. Schneider, and I. Bloch, Observation of many-body localization of interacting fermions in a quasirandom optical lattice, Science 349, 842 (2015).

[8] J.-y. Choi, S. Hild, J. Zeiher, P. Schauß, A. Rubio-Abadal, T. Yefsah, V. Khemani, D. A. Huse, I. Bloch, and C. Gross, Exploring the many-body localization transition in two dimensions, Science 352, 1547 (2016).

[9] J. Smith, A. Lee, P. Richerme, B. Neyenhuis, P. W. Hess, P. Hauke, M. Heyl, D. A. Huse, and C. Monroe, Many-body localization in a quantum simulator with programmable random disorder, Nat. Phys. 12, 907 (2016).

[10] H. Bernien, S. Schwartz, A. Keesling, H. Levine, A. Omran, H. Pichler, S. Choi, A. S. Zibrov, M. Endres, M. Greiner, V. Vuletić, and M. D. Lukin, Probing many-body dynamics on a 51-atom quantum simulator, Nature 551, 579 (2017).

[11] C. J. Turner, A. A. Michailidis, D. A. Abanin, M. Serbyn, and Z. Papić, Weak ergodicity breaking from quantum many-body scars, Nat. Phys. 14, 745 (2018).

32 [12] P. Barmettler, D. Poletti, M. Cheneau, and C. Kollath, Propagation front of correlations in an interacting bose gas, Phys. Rev. A 85, 053625 (2012).

[13] K. Nagao, M. Kunimi, Y. Takasu, Y. Takahashi, and I. Danshita, Semiclassical quench dynamics of bose gases in optical lattices, Phys. Rev. A 99, 023622 (2019).

[14] K. Nagao, Y. Takasu, Y. Takahashi, and I. Danshita, Su(3) truncated wigner approximation for strongly interacting bose gases, arXiv:2008.09900 .

238 [15] A. Mokhtari-Jazi, M. R. C. Fitzpatrick, and M. P. Kennett, Phase and group velocities for correlation spreading in the mott phase of the bose-hubbard model in dimensions greater than one, Phys. Rev. A 103, 023334 (2021).

241 [16] M. Greiner, O. Mandel, T. Esslinger, T. W. Hänsch, and I. Bloch, Quantum phase transition from a superfluid to a mott insulator in a gas of ultracold atoms, Nature 415, 39 (2002).

243 [17] A. M. Läuchli and C. Kollath, Spreading of correlations and entanglement after a quench in the one-dimensional bose-hubbard model, J. Stat. Mech. Theory Exp. 2008, P05018 (2008). 
245 [18] N. Schuch, S. K. Harrison, T. J. Osborne, and J. Eisert, Information propagation for $246 \quad$ interacting-particle systems, Phys. Rev. A 84, 032309 (2011).

247 [19] G. Carleo, F. Becca, L. Sanchez-Palencia, S. Sorella, and M. Fabrizio, Light-cone effect and

250 [20] L. Cevolani, J. Despres, G. Carleo, L. Tagliacozzo, and L. Sanchez-Palencia, Universal scaling

260 [24] T. Kuwahara and K. Saito, Lieb-robinson bound and almost-linear light cone in interacting supersonic correlations in one- and two-dimensional bosonic superfluids, Phys. Rev. A 89, 031602(R) (2014). laws for correlation spreading in quantum systems with short- and long-range interactions, Phys. Rev. B 98, 024302 (2018).

[21] M. R. C. Fitzpatrick and M. P. Kennett, Light-cone-like spreading of single-particle correlations in the bose-hubbard model after a quantum quench in the strong-coupling regime, Phys. Rev. A 98, 053618 (2018).

[22] J. Despres, L. Villa, and L. Sanchez-Palencia, Twofold correlation spreading in a strongly correlated lattice bose gas, Sci. Rep. 9, 4135 (2019). boson systems, Phys. Rev. Lett. 127, 070403 (2021).

[25] M. A. Martín-Delgado, M. Roncaglia, and G. Sierra, Stripe ansätze from exactly solved models, Phys. Rev. B 64, 075117 (2001).

[26] F. Verstraete and J. I. Cirac, Renormalization algorithms for quantum-many body systems in two and higher dimensions, arXiv:cond-mat/0407066 .

[27] F. Verstraete and J. I. Cirac, Valence-bond states for quantum computation, Phys. Rev. A 70, 060302(R) (2004).

[28] F. Verstraete, V. Murg, and J. I. Cirac, Matrix product states, projected entangled pair states, and variational renormalization group methods for quantum spin systems, Adv. Phys. 57, 143 (2008).

271 [29] J. Jordan, R. Orús, G. Vidal, F. Verstraete, and J. I. Cirac, Classical simulation of infinite-size 272 quantum lattice systems in two spatial dimensions, Phys. Rev. Lett. 101, 250602 (2008).

273 [30] H. N. Phien, J. A. Bengua, H. D. Tuan, P. Corboz, and R. Orús, Infinite projected entangled $274 \quad$ pair states algorithm improved: Fast full update and gauge fixing, Phys. Rev. B 92, 035142 (2015). 
276 [31] R. Orús, A practical introduction to tensor networks: Matrix product states and projected $277 \quad$ entangled pair states, Ann. Phys. (N.Y.) 349, 117 (2014).

278 [32] R. Orús, Tensor networks for complex quantum systems, Nat. Rev. Phys. 1, 538 (2019).

279 [33] Y. Hieida, K. Okunishi, and Y. Akutsu, Numerical renormalization approach to two280 dimensional quantum antiferromagnets with valence-bond-solid type ground state, New J. Phys. $281 \quad 1,7(1999)$.

282 [34] K. Okunishi and T. Nishino, Kramers-wannier approximation for the 3d ising model, Prog. $283 \quad$ Theor. Phys. 103, 541 (2000).

284 [35] T. Nishino, Y. Hieida, K. Okunishi, N. Maeshima, Y. Akutsu, and A. Gendiar, Two285 dimensional tensor product variational formulation, Prog. Theor. Phys. 105, 409 (2001).

286 [36] N. Maeshima, Y. Hieida, Y. Akutsu, T. Nishino, and K. Okunishi, Vertical density matrix al287 gorithm: A higher-dimensional numerical renormalization scheme based on the tensor product $288 \quad$ state ansatz, Phys. Rev. E 64, 016705 (2001).

289 [37] Y. Nishio, N. Maeshima, A. Gendiar, and T. Nishino, Tensor product variational formulation 290 for quantum systems, arXiv:cond-mat/0401115 .

291 [38] A. Kshetrimayum, H. Weimer, and R. Orús, A simple tensor network algorithm for two292 dimensional steady states, Nat. Commun. 8, 1 (2017).

293 [39] P. Czarnik, J. Dziarmaga, and P. Corboz, Time evolution of an infinite projected entangled $294 \quad$ pair state: An efficient algorithm, Phys. Rev. B 99, 035115 (2019).

295 [40] C. Hubig and J. I. Cirac, Time-dependent study of disordered models with infinite projected $296 \quad$ entangled pair states, SciPost Phys. 6, 31 (2019).

297 [41] A. Kshetrimayum, M. Goihl, and J. Eisert, Time evolution of many-body localized systems in $298 \quad$ two spatial dimensions, Phys. Rev. B 102, 235132 (2020).

299 [42] A. Kshetrimayum, M. Goihl, D. M. Kennes, and J. Eisert, Quantum time crystals with pro$300 \quad$ grammable disorder in higher dimensions, Phys. Rev. B 103, 224205 (2021).

301 [43] C. Hubig, A. Bohrdt, M. Knap, F. Grusdt, and J. I. Cirac, Evaluation of time-dependent 302 correlators after a local quench in ipeps: hole motion in the t-j model, SciPost Phys. 8, 21 $303 \quad(2020)$.

304 [44] Álvaro M. Alhambra and J. I. Cirac, Locally accurate tensor networks for thermal states and 305 time evolution, arXiv:2106.00710 . 
306 [45] M. Schmitt, M. M. Rams, J. Dziarmaga, M. Heyl, and W. H. Zurek, Quantum phase transition $307 \quad$ dynamics in the two-dimensional transverse-field ising model, arXiv:2106.09046 .

308 [46] J. Dziarmaga, Time evolution of an infinite projected entangled pair state: Neighborhood tensor $309 \quad$ update, Phys. Rev. B 104, 094411 (2021).

310 [47] H. Weimer, A. Kshetrimayum, and R. Orús, Simulation methods for open quantum many-body $311 \quad$ systems, Rev. Mod. Phys. 93, 015008 (2021).

312 [48] C. Mc Keever and M. H. Szymańska, Stable ipepo tensor-network algorithm for dynamics of 313 two-dimensional open quantum lattice models, Phys. Rev. X 11, 021035 (2021).

314 [49] D. Kilda, A. Biella, M. Schiro, R. Fazio, and J. Keeling, On the stability of the infinite Projected 315 Entangled Pair Operator ansatz for driven-dissipative 2D lattices, SciPost Phys. Core 4, 5 316 (2021).

317 [50] M. P. A. Fisher, P. B. Weichman, G. Grinstein, and D. S. Fisher, Boson localization and the 318 superfluid-insulator transition, Phys. Rev. B 40, 546 (1989).

319 [51] D. Jaksch, C. Bruder, J. I. Cirac, C. W. Gardiner, and P. Zoller, Cold bosonic atoms in optical $320 \quad$ lattices, Phys. Rev. Lett. 81, 3108 (1998).

321 [52] P. Weinberg and M. Bukov, QuSpin: a Python Package for Dynamics and Exact Diagonali322 sation of Quantum Many Body Systems part I: spin chains, SciPost Phys. 2, 003 (2017).

323 [53] P. Weinberg and M. Bukov, QuSpin: a Python Package for Dynamics and Exact Diagonalisation of Quantum Many Body Systems. Part II: bosons, fermions and higher spins, SciPost $325 \quad$ Phys. 7, 20 (2019).

326 [54] See Supplemental Materials.

327 [55] E. H. Lieb and D. W. Robinson, The finite group velocity of quantum spin systems, Commun. $328 \quad$ Math. Phys. 28, 251 (1972).

329 [56] M. B. Hastings, Locality in quantum systems, arXiv:1008.5137 .

330 [57] N. Elstner and H. Monien, Dynamics and thermodynamics of the bose-hubbard model, Phys. 331 Rev. B 59, 12184 (1999).

332 [58] B. Capogrosso-Sansone, Ş. G. Söyler, N. Prokof'ev, and B. Svistunov, Monte carlo study of 333 the two-dimensional bose-hubbard model, Phys. Rev. A 77, 015602 (2008).

334 [59] K. V. Krutitsky, Ultracold bosons with short-range interaction in regular optical lattices, Phys. $335 \quad$ Rep. 607, 1 (2016). 
336 [60] S. D. Huber, E. Altman, H. P. Büchler, and G. Blatter, Dynamical properties of ultracold $337 \quad$ bosons in an optical lattice, Phys. Rev. B 75, 085106 (2007).

338 [61] S. M. Davidson and A. Polkovnikov, su(3) semiclassical representation of quantum dynamics $339 \quad$ of interacting spins, Phys. Rev. Lett. 114, 045701 (2015).

340 [62] V. Murg, F. Verstraete, and J. I. Cirac, Variational study of hard-core bosons in a two$341 \quad$ dimensional optical lattice using projected entangled pair states, Phys. Rev. A 75, 033605 342 (2007).

343 [63] J. Jordan, R. Orús, and G. Vidal, Numerical study of the hard-core bose-hubbard model on an $344 \quad$ infinite square lattice, Phys. Rev. B 79, 174515 (2009).

345 [64] A. Kshetrimayum, M. Rizzi, J. Eisert, and R. Orús, Tensor network annealing algorithm for 346 two-dimensional thermal states, Phys. Rev. Lett. 122, 070502 (2019).

347 [65] S. S. Jahromi and R. Orús, Universal tensor-network algorithm for any infinite lattice, Phys. $348 \quad$ Rev. B 99, 195105 (2019).

349 [66] S. S. Jahromi and R. Orús, Thermal bosons in 3d optical lattices via tensor networks, Sci. $350 \quad$ Rep. 10, 19051 (2020).

351 [67] P. Schmoll, S. S. Jahromi, M. Hörmann, M. Mühlhauser, K. P. Schmidt, and R. Orús, Fine 352 grained tensor network methods, Phys. Rev. Lett. 124, 200603 (2020).

353 [68] W.-L. Tu, H.-K. Wu, and T. Suzuki, Frustration-induced supersolid phases of extended $354 \quad$ bose-hubbard model in the hard-core limit, J. Phys.: Cond. Mat. 32, 455401 (2020).

355 [69] H.-K. Wu and W.-L. Tu, Competing quantum phases of hard-core bosons with tilted dipole356 dipole interaction, Phys. Rev. A 102, 053306 (2020).

357 [70] P. C. G. Vlaar and P. Corboz, Simulation of three-dimensional quantum systems with projected $358 \quad$ entangled-pair states, Phys. Rev. B 103, 205137 (2021).

359 [71] H. F. Trotter, On the product of semi-groups of operators, Proc. Amer. Math. Soc. 10, 545 $360 \quad(1959)$.

361 [72] M. Suzuki, Pair-product model of heisenberg ferromagnets, J. Phys. Soc. Jpn. 21, 2274 (1966).

362 [73] M. Suzuki, Relationship between d-dimensional quantal spin systems and $(d+1)$-dimensional ising systems: Equivalence, critical exponents and systematic approximants of the partition 364 function and spin correlations, Prog. Theor. Phys. 56, 1454 (1976).

365 [74] H. C. Jiang, Z. Y. Weng, and T. Xiang, Accurate determination of tensor network state of 366 quantum lattice models in two dimensions, Phys. Rev. Lett. 101, 090603 (2008). 
367 [75] TeNeS: https://github.com/issp-center-dev/tenes.

368 [76] pTNS: https://github.com/tsuyoshiokubo/ptns.

369 [77] T. Nishino and K. Okunishi, Corner transfer matrix renormalization group method, J. Phys. $370 \quad$ Soc. Jpn. 65, 891 (1996).

371 [78] T. Nishino and K. Okunishi, Corner transfer matrix algorithm for classical renormalization $372 \quad$ group, J. Phys. Soc. Jpn. 66, 3040 (1997).

373 [79] T. Nishino, T. Hikihara, K. Okunishi, and Y. Hieida, Density matrix renormalization group: $374 \quad$ Introduction from a variational point of view, Int. J. Mod. Phys. B 13, 1 (1999).

375 [80] R. Orús and G. Vidal, Simulation of two-dimensional quantum systems on an infinite lattice 376 revisited: Corner transfer matrix for tensor contraction, Phys. Rev. B 80, 094403 (2009).

377 [81] P. Corboz, J. Jordan, and G. Vidal, Simulation of fermionic lattice models in two dimensions 378 with projected entangled-pair states: Next-nearest neighbor hamiltonians, Phys. Rev. B 82, $379 \quad 245119(2010)$.

380 [82] P. Corboz, S. R. White, G. Vidal, and M. Troyer, Stripes in the two-dimensional t-j model $381 \quad$ with infinite projected entangled-pair states, Phys. Rev. B 84, 041108(R) (2011).

382 [83] P. Corboz, T. M. Rice, and M. Troyer, Competing states in the t-j model: Uniform d-wave $383 \quad$ state versus stripe state, Phys. Rev. Lett. 113, 046402 (2014).

\section{$384 \quad$ ACKNOWLEDGMENTS}

385 We acknowledge fruitful discussions with S. Goto and K. Nagao. We thank Y. Takahashi 386 and Y. Takasu for useful discussions and the experimental data. This work was financially 387 supported by JSPS KAKENHI (Grants Nos. JP18H05228, JP21H01014, and JP21K13855), 388 by JST CREST (Grant No. JPMJCR1673), by JST FOREST (Grant No. JPMJFR202T), 389 and by MEXT Q-LEAP (Grant No. JPMXS0118069021). The numerical computations were 390 performed on computers at the Yukawa Institute Computer Facility and on computers at 391 the Supercomputer Center, the Institute for Solid State Physics, the University of Tokyo.

\section{VII. AUTHOR CONTRIBUTIONS}

393 R.K. and I.D. designed and coordinated the studies. R.K. performed the numerical 394 simulations. R.K. and I.D. contributed to the writing of the manuscript. 


\section{VIII. COMPETING INTERESTS}

396 The authors declare no competing interests.

${ }_{397}$ IX. ADDITIONAL INFORMATION

398 Supplementary information is available for this paper at XXXXXX. 


\section{Supplementary Files}

This is a list of supplementary files associated with this preprint. Click to download.

- supp.pdf 